\title{
Polarization Sensitive Optical Coherence Tomography of the Rabbit Eye
}

\author{
Mathieu G. Ducros, Johannes F. de Boer, Huai-En Huang, Lawrence C. Chao, Zhongping Chen, \\ J. Stuart Nelson, Thomas E. Milner, and H. Grady Rylander, III, Associate Member, IEEE
}

\begin{abstract}
A polarization-sensitive optical coherence tomography (PSOCT) system was used to acquire depth-resolved images of the Stokes parameters of light backreflected from ex vivo rabbit eyes. The light backreflected from the eye interferes with that from the reference arm and is coherently detected in two orthogonal polarization channels. The two signals are digitized and the four Stokes parameters $(I, Q, U$, and $V)$ of the backreflected light are computed for light backreflected from each longitudinal/lateral position in the eye. From the measured Stokes parameters, an estimate of the relative phase retardation between the two orthogonal polarizations can be determined. Two eyes were enucleated, imaged within 6-h postmortem and histology performed. Images of the Stokes parameters of light backreflected from the corneal stroma show significant local variations in the polarization state, possibly due to local changes in stromal structure. Depth-resolved Stokes parameter images of light backreflected from the retina were also acquired. A birefringent layer was observed at the position consistent with the known location of the nerve fiber layer (NFL). The local thickness of the birefringent layer determined with PSOCT was in good agreement with values determined histologically.
\end{abstract}

Index Terms - Biomedical optical imaging, birefringence, cornea, glaucoma, interferometry, optical tomography, polarimetry, retina.

\section{INTRODUCTION}

A NUMBER of biological tissues such as muscle, tendons and cornea are known to be birefringent. Birefringence in biological media is frequently due to the presence of asymmetrical structures of a given index of refraction (for example, collagen fibrils, microtubules, actin, and myosin filaments) dispersed in an oriented manner in a medium of different index of refraction (for example, cytoplasm, extracellular fluid). This property, known as form birefringence, is directly related to

Manuscript received February 4, 1999. This work was supported by the Institute of Arthritis, Musculoskeletal, and Skin Diseases under Research Grant 1R29-AR41638-01A1 and Research Grant 1R01-AR42437-01A1, by the Whitaker Foundation under Grant 23281, by the National Center for Research Resources at the National Institutes of Health (Laser Microbeam and Medical Program, RR-01192), by the U.S. Department of Energy under Contract DE-FG03-91ER61227, by the Office of Naval Research under Contract N00014-94-1-0874, by an Endowment from the Beckman Laser Institute, by the Heart, Lung, and Blood Institute at the National Institutes of Health under Research Grant R01 HL59472-02, and by the Air Force Office of Scientific Research through MURI from DDR\&E Contract F4962098-1-0480.

M. G. Ducros, T. E. Milner, and H. G. Rylander, III, are with the Biomedical Engineering Program, University of Texas at Austin, Austin, TX 78712 USA.

J. F. de Boer, H. Huang, L. C. Chao, Z. Chen, and J. S. Nelson are with the Beckman Laser Institute and Medical Clinic, University of California-Irvine, Irvine, CA 92612 USA.

Publisher Item Identifier S 1077-260X(99)07574-7. tissue structure and organization. Molecular conformation is a second origin of birefringence in biological media and is identical to that in crystals.

The objective of this work is to observe birefringence in the eye using polarization-sensitive optical coherence tomography (PSOCT). With this technique we are able to acquire four polarization images corresponding to the four Stokes parameters of light backreflected from any position in the tissue. In the present work, PSOCT images were acquired in the cornea and retina of ex vivo rabbit eyes.

In the eye the corneal stroma and the retinal nerve fiber layer (NFL) are birefringent. The crystalline lens is also most likely birefringent, but will not be discussed in this paper.

The corneal stroma is the central and thickest layer of the cornea. It consists of great number of stacked lamellae. Each lamella has the form of a thin (a few microns thick) ribbon. Principal constituents are collagen fibrils, glycosaminoglycans and water. The fibrils are oriented parallel to the surfaces of the lamellae. Due to this particular structure, a lamella can be compared to a linear retarder in which slow and fast axes lie, respectively, parallel and perpendicular to the fibril direction. Each lamella induces a small phase retardation in incoming polarized light. Experimental and numerical studies have been conducted to determine the possible structure of the corneal stroma. Some investigators suggested that the fibers in successive lamellae have totally random orientation [1], while others believed that the lamella orientation is not totally random and that a preferential direction exists [2]-[4]. Most studies on corneal stroma structure have been conducted using polarization microscopy of excised cornea. The local phase retardation (due to birefringence) and optic axis of light transmitted through the whole cornea can be measured with the sample placed in a system of crossedpolarizers and compensator plates. Using PSOCT we can measure the change in polarization state of light backreflected from a given position while the cornea remains in situ. Therefore, PSOCT can be used to investigate the corneal stroma structure. Furthermore, depth resolved polarization images in the cornea may be useful to observe in vivo thermal damage that could occur in treatments such as photo-refractive keratectomy (PRK) and laser in situ keratomileusis (LASIK). In this paper, we present and interpret a PSOCT cross-sectional image obtained in the cornea of enucleated rabbit eye.

The NFL is the most anterior layer of the retina and consists of glial cells and axons of ganglion cells. The axons converge radially to the optic nerve where they exit the 
ocular globe. The NFL can be up to $400 \mu \mathrm{m}$ thick at the edge of the optic nerve [5]. The NFL birefringence is due to the dense parallel arrangement of axon membranes and microtubules inside the axons [6], [7]. Glaucoma is the second leading cause of blindness worldwide and affects the NFL. In glaucoma, the retinal ganglion cells die and the axons in the NFL disappear. This process is irreversible. Quigley et al. examined postmortem histological sections of optic nerve heads in glaucomatous and normal patients [8]. They found that the total axon count drops from about 1 million for normal subjects to about 1000 in advanced glaucoma cases. They also demonstrated that up to $40 \%$ of the ganglion cell axons may be lost before a reduction in visual field sensitivity is detected. An instrument providing a precise and reliable measurement of the NFL thickness and structural damage could greatly improve detection of glaucoma method and aid in follow-up examinations.

Conventional optical coherence tomography (OCT) and scanning laser polarimetry (SLP) are two experimental techniques being investigated to measure accurately the NFL thickness and to differentiate between glaucomatous and normal conditions [9], [10]. A commercially available OCT system (Humphrey Instruments, San Leandro, CA) provides a cross-sectional image of the retina around the optic nerve with a depth resolution on the order of $15 \mu \mathrm{m}$. The pixel brightness in an OCT image is proportional to the intensity of the light backreflected from the corresponding tissue element [11], [12]. Therefore anatomical layers with significantly different optical properties can be differentiated. Toth et al. compared OCT and histological images recorded from the same locations in the retinas of primates [13]. They concluded that regions of high reflectivity correspond to horizontally aligned components such as the NFL as well as to the pigment epithelium and choroid. Schuman et al. evaluated the reproducibility of NFL thickness measurement with OCT [9]. They developed an image processing algorithm to measure the NFL thickness at 12 retinal positions on a circle centered around the optic nerve. The standard deviation of measured NFL thickness at each point (for multiple measurements on the same patient) varied from $18-34 \mu \mathrm{m}$. The measured NFL thickness is about $150 \mu \mathrm{m}$ for normal patients and $100 \mu \mathrm{m}$ for glaucoma patients. From this study, the investigators concluded that use of OCT may allow differentiation between normal and advanced glaucoma patients. Detecting the onset of glaucoma and following evolution of the disease in a patient using conventional OCT appears more problematic.

SLP provides an indirect measurement of the NFL thickness. The eye is illuminated with polarization-modulated laser light and a scanning laser beam is focused onto the retina. The polarization state of light passing through the birefringent NFL is altered. A portion the incident light propagates through the NFL, is backreflected from the retina, exits the eye, and is detected. The phase retardation between two orthogonal polarization components is measured. The NFL thickness is assumed to be proportional to the measured retardation. The SLP principle was first demonstrated by Weinreb using two fixed monkey eyes [14]. He found a significant correlation between the measured phase retardation and the NFL thickness determined histologically (7.4 $\mu \mathrm{m}$ per degree of retardation). Dreher and colleagues found that the birefringent fast axis of the NFL is arranged radially with respect to the optic nerve head, which is in good agreement with observations [15].

In a recent study, Weinreb et al. evaluated the sensitivity and specificity of a commercialized scanning laser polarimeter (Laser Diagnostic Technologies, San Diego, CA) for glaucoma diagnostics [16]. They optimized the data analysis algorithm and found a sensitivity of $74 \%$ and a specificity of $92 \%$. In other published works [17], [18], however, high intersubject and significant intrasubject variabilities in local retardation measurements have been reported. Therefore, NFL thickness measurement with SLP is not widely accepted by the ophthalmologic community as a reliable glaucoma diagnostic method. The numerical aperture of the eye limits the SLP longitudinal resolution to 200-300 $\mu \mathrm{m}$. The retardation measured with SLP is an average value of all light paths backreflected from the confocal region. The measurement reliability of SLP would likely be improved with a better longitudinal resolution. Because PSOCT combines the advantages of OCT (high depth resolution) and SLP (detection of tissue birefringence) recorded images may provide a better contrast between the birefringent NFL and other retinal structures. In this paper we demonstrate the possibility to measure the NFL thickness in ex vivo rabbit retina using a PSOCT system.

\section{METHOD}

\section{A. PSOCT Principle and Setup}

PSOCT is a newly developed biomedical imaging technique able to measure the polarization state of light backreflected from a given depth and lateral position within a sample. PSOCT imaging of birefringence has been demonstrated in bovine tendon [19] and porcine myocardium [20]. Reduction in birefringence due to thermal damage was also observed with PSOCT in porcine tendons, skin [21] and myocardium [22]. The PSOCT technique is particularly attractive for clinical applications such as in vivo burn depth determination and real time laser thermal damage assessment.

The PSOCT system used to acquire the images presented in this paper is a polarization sensitive Michelson interferometer (Fig. 1). The source, a super luminescent diode (SLD), emits $0.8 \mathrm{~mW}$ in air at $856 \mathrm{~nm}$. Full-width at half-maximum (FWHM) spectral bandwidth is $25 \mathrm{~nm}$. The SLD output is collimated and linearly polarized horizontally, then divided in two equal intensity beams in the sample and reference arms by a nonpolarizing beam splitter (BS). A zeroth-order quarter wave (QWP1) is placed in the reference arm with its optic axis oriented at $22.5^{\circ}$ with respect to the horizontal. After reflection from the reference mirror and a return passage through QWP1 the reference light is linearly polarized at $45^{\circ}$ and is equally split over both detectors by a polarizing beam splitter PBS. In the sample arm, quarter wave plate QWP2 oriented at $45^{\circ}$ to the horizontal transforms the light into a right circularly polarized state. The sample illumination beam is focused to a $17-\mu \mathrm{m}$ diameter spot. Light backreflected from the sample and reference mirror interfere only if the optical pathlength in each 


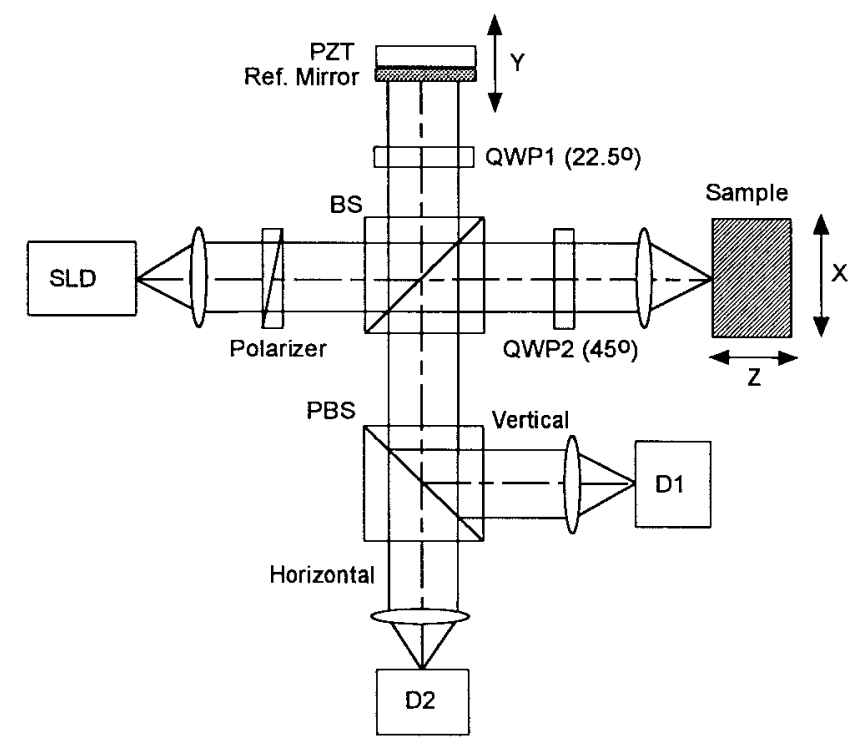

Fig. 1. PSOCT system schematic. The short coherence source (SLD) illuminates a Michelson interferometer setup. The source light is collimated, horizontally polarized, and split into reference and sample beams by a nonpolarizing beam splitter (BS). In the reference arm, light is reflected by the reference mirror mounted on a PZT oscillator. The PZT displacement creates a $100 \mathrm{~Hz}$ saw-tooth path length modulation of amplitude $20 \mu \mathrm{m}$, inducing a light Doppler shift of about $6 \mathrm{kHz}$. After double passage in a quarter wave plate at $22.5^{\circ}$ (QWP1), the reference light is linearly polarized at $45^{\circ}$. In the sample arm, light is circularly polarized by the quarter wave plate at $45^{\circ}$ (QWP2) and focused to a 17- $\mu$ m-diameter spot on the sample. Light backreflected from the sample is in an unknown elliptical polarization state. Interference with reference light takes place only if the difference in optical path between the sample and reference light is smaller than the coherence length of the SLD. In the detection arm, the light is divided into two orthogonal polarization components by the polarizing beam splitter PBS and focused onto the detectors D1 and D2.

arm is equal to within the source coherence length. The light is then divided into two orthogonal polarization components by PBS. The vertical and horizontal polarization components are focused onto detectors D1 and D2, respectively. The PZT moves the reference mirror with a saw-tooth waveform of amplitude $20 \mu \mathrm{m}$ and frequency $100 \mathrm{~Hz}$ giving an interference fringe carrier frequency of approximately $6 \mathrm{KHz}$. Signals detected by D1 and D2 are amplified, bandpass filtered at the carrier frequency and sampled simultaneously. The Stokes parameters for the light backreflected from the sample are calculated using interference signals over the central $10-\mu \mathrm{m}$ displacement of the PZT [23]. The sample is displaced laterally ( $X$ stage) at $1 \mathrm{~mm} / \mathrm{s}$ with respect to the illumination optics. To scan in depth the sample beam focus is displaced ( $Z$ stage) in steps of $10 \mu \mathrm{m}$ and the reference arm is moved to overlap the coherence gate and beam focus position ( $Y$ stage). Thus the pixel dimensions in the images of the Stokes parameters is $10 \mu \mathrm{m} \times 10 \mu \mathrm{m}$.

Coherent detection of the amplitude and phase of the interference fringes in orthogonal polarization channels allows for computation of the Stokes parameters [23]. Therefore, the polarization state of the backreflected light is completely determined. In previous PSOCT systems, only the phase retardation was assessed by computing the ratio of the interference fringe envelope detected in orthogonal polarization channels.

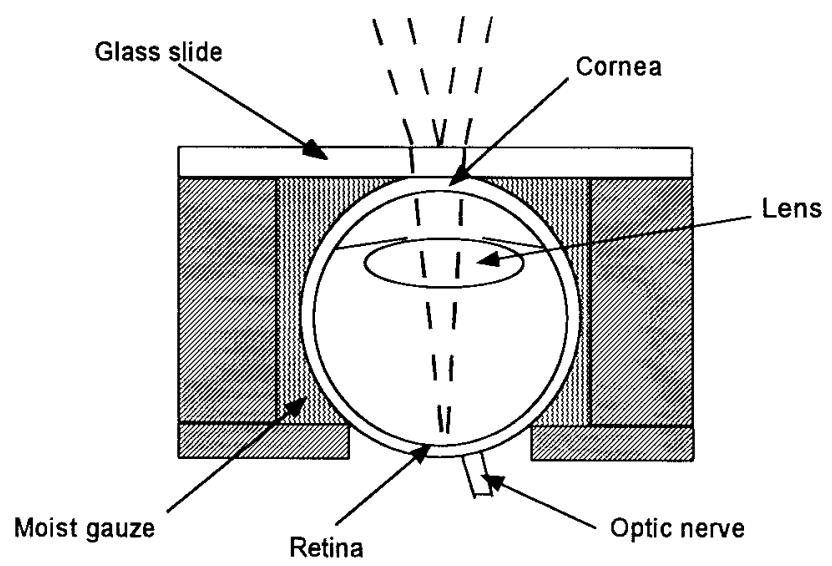

Fig. 2. Eye holder. The sample was surrounded by moist gauze. A flat glass slide was placed on the cornea to allow good visualization of the eye fundus. For imaging the cornea, the beam focus was placed a few hundred micrometers above the glass slide at the beginning of the scan. For imaging the retina, the eye was oriented to place the optic nerve in the field of view and the beam focus was positioned above the vitreous-retinal interface.

The four Stokes parameters are denoted as $I, Q, U$, and $V$. The $I$ parameter represents the total light intensity $\left(I_{t}\right)$ backreflected from the eye at each position and is independent of the polarization state:

$$
I=I_{t}
$$

$Q$ represents the difference between the intensities of light linearly polarized at $0^{\circ}\left(I_{0^{\circ}}\right)$ and $90^{\circ}\left(I_{90^{\circ}}\right)$, normalized to the total intensity:

$$
Q=\frac{I_{0^{\circ}}-I_{90^{\circ}}}{I_{t}} .
$$

$U$ represents the difference in intensities of light linearly polarized at $+45^{\circ}\left(I_{+45^{\circ}}\right)$ and $-45^{\circ}\left(I_{-45^{\circ}}\right)$ normalized to the total intensity:

$$
U=\frac{I_{+45^{\circ}}-I_{-45^{\circ}}}{I_{t}} .
$$

$V$ represents the difference in intensities of right $\left(I_{r c}\right)$ and left $\left(I_{l c}\right)$ circular polarized light, normalized to the total intensity:

$$
V=\frac{I_{r c}-I_{l c}}{I_{t}}
$$

Gray scale images of the $I, Q, U$, and $V$ Stokes parameters are presented. In the $I$ image pixel brightness is proportional to the local value of $I$ in $\mathrm{dB}$. In images of $Q, U$, and $V$, pixel brightness varies between white for +1 and black for -1 .

We also calculated for each pixel the parameter $\Phi$ defined by:

$$
\Phi=\arctan \left(\frac{\sqrt{Q^{2}+U^{2}}}{-V}\right) .
$$

In the Poincare sphere representation [24], $\Phi$ is the angle between the Stokes vectors of light incident on the eye ( $Q=0, U=0, V=1$ ) and that backreflected from a given tissue position. According to the known structure of the corneal stroma (stack of lamellae acting as linear retarders 

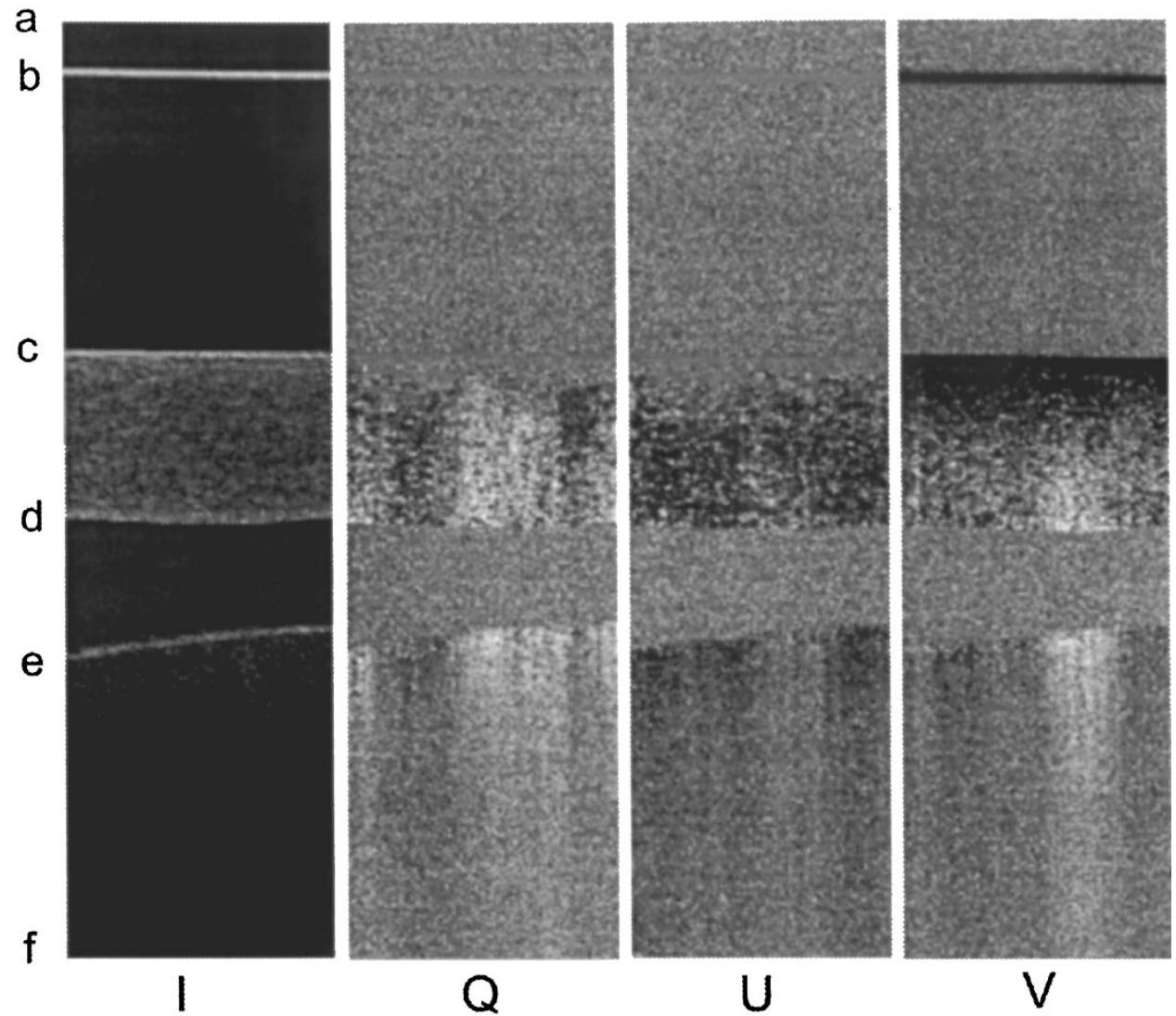

Fig. 3. From left to right: $I, Q, U$, and $V$ Stokes parameters images in the rabbit cornea. Each image is $1 \mathrm{~mm}$ wide $\times 3.5 \mathrm{~mm}$ deep. The pixel size is $10 \times 10 \mu \mathrm{m}$. The letters a and $\mathrm{f}$ correspond to the beginning and end of the scan and $\mathrm{b}$, c, $\mathrm{d}$ and e indicates the air-glass, glass-cornea, cornea-aqueous, aqueous-crystalline lens interfaces. Five zones can be seen from top to bottom: air (a-b), glass slide (b-c), cornea (c-d), anterior chamber (d-e), and part of the crystalline lens (e-f). In the cornea, the $I$ image is relatively uniform, but the $Q, U$, and $V$ images show regions where the backreflected light has different polarization states indicating structural differences in the corneal stroma.

parallel to the surface of the cornea) and retinal NFL (axons membranes and microtubules running parallel to the surface of the retina), these tissues can be viewed as linear retarders with fast and slow optic axes perpendicular to the light propagation direction (the illumination beam being perpendicular to the cornea surface). In these conditions, $\Phi$ represents the phase retardation between two orthogonal polarization components of light backreflected from a given tissue element inside the eye relative to the phase retardation between two orthogonal components of light incident onto the eye. $\Phi$ equals $0^{\circ}$ for left circularly polarized light, $90^{\circ}$ for linearly polarized light and $180^{\circ}$ for right circularly polarized light. When the backreflected light is unpolarized, or when the intensity is lower than the system noise level, $\Phi$ varies randomly between $0^{\circ}$ and $180^{\circ}$ and averages $90^{\circ}$.

\section{B. Samples}

A New Zealand white rabbit was sacrificed and the eyes enucleated and placed in a chilled saline solution. Images were acquired less than 6-h post mortem. The sample holder is diagramed in Fig. 2. The entry window of the eye holder was constructed of a flat glass slide that was gently pressed against the cornea to reduce the refractive power. This was extremely helpful to visualize the eye fundus and to maintain the eye in a stable position during the measurements. For imaging the cornea, the beam focus was placed approximately at the apex of the cornea. For imaging the retina, the beam focus was placed approximately on the superior and/or inferior region of the optic nerve head where the NFL is the thickest. A red aiming beam, aligned with the broadband infrared SLD beam, was used to determine the imaging position on the retina. The red light was partially transmitted through the choroid and sclera, and visible on the back side of the eye. A black ink dot was placed on the back of the eye at the lateral edges of the imaged region to locate the corresponding area in histology. After measurements, the eye was fixed in formalin and histology performed.

\section{RESUlTS AND DisCUSSION}

\section{A PSOCT Imaging in the Cornea}

1) Stokes Parameter Images: Fig. 3 presents the Stokes parameter images (from left to right $I, Q, U$, and $V$ ) acquired in the cornea of an enucleated rabbit eye positioned in the sample holder. Each image is $1 \mathrm{~mm}$ wide $\times 3.5 \mathrm{~mm}$ deep.

The letters to the left of the $I$ image refer to: a) the beginning of the scan, b) the air-glass interface, c) the glass-cornea interface, d) the cornea-aqueous interface, e) the aqueous-crystalline lens interface, and $f$ ) the end of the scan. As expected, the glass slide $(b-c)$, is transparent 


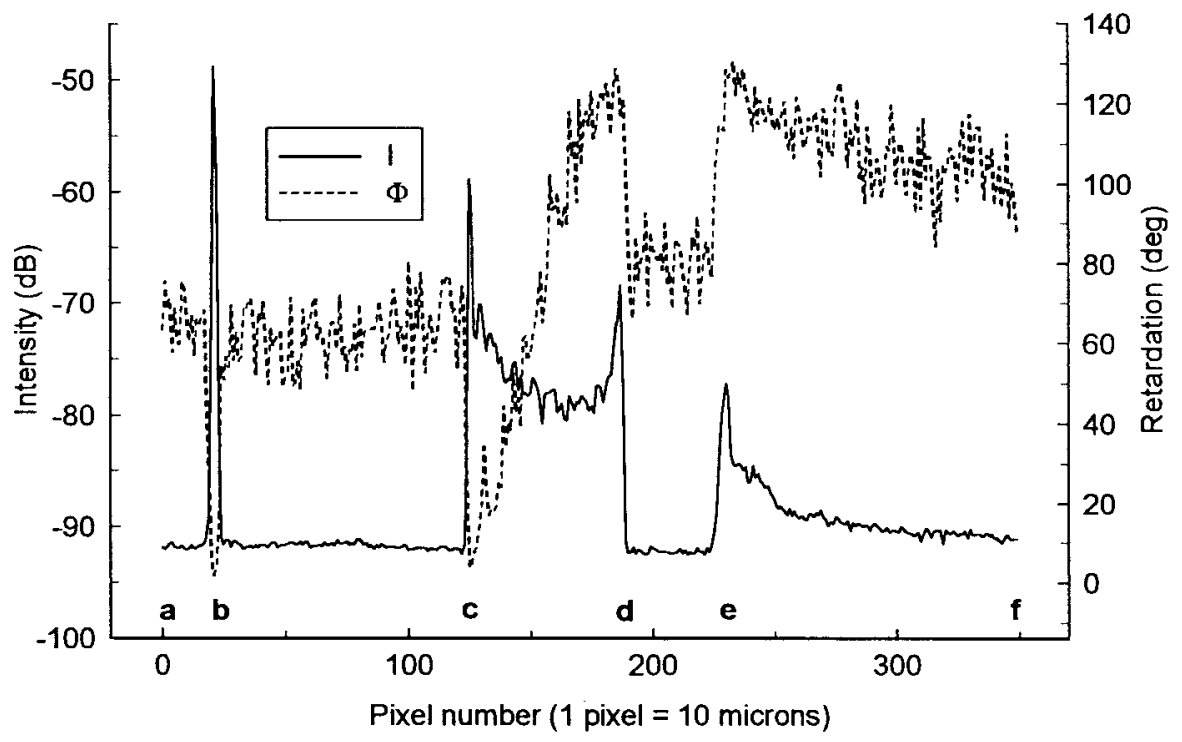

Fig. 4. Plots of $I$ and $\Phi$ parameters versus depth. The letters a, b, c, d, e, and f indicate the depth positions introduced on Fig. 3. Plots were calculated by averaging 25 adjacent A-scans of the $I$ and $\Phi$ parameter images. $\Phi$ was calculated according to (5). At all interfaces a peak of intensity ( $I$ ) can be observed. In the air, the glass and the aqueous no light is backreflected and $I$ is about $-92 \mathrm{~dB}$ and $\Phi 65^{\circ}$. In the cornea $I$ varies between -70 and $-80 \mathrm{~dB}$ and in the crystalline lens it slowly drops down to the noise level. At the front and back glass slide surfaces $\mathrm{F}$ drops to almost $0^{\circ}$. In the cornea $(\mathrm{c}-\mathrm{d})$ it increases from $0^{\circ}$ to $120^{\circ}$. It is still $120^{\circ}$ at the aqueous-lens interface, showing that the aqueous did not affect the $\Phi$ parameter value. In the anterior part of the crystalline lens it decreases from $120^{\circ}$ to $100^{\circ}$.

( $I$ minimum) and does not induce any change on the light polarization. $Q, U$, and $V$ appear gray. Their average value is 0 and variations are due only to noise. The front and back specularly reflective surfaces of the glass appear bright in the $I$ image, black in the $V$ image, and gray in the $Q$ and $U$ images, meaning that the backreflected light polarization is left circular. The helicity of the incident light is reversed as expected for specular reflection. In the cornea (about $0.6 \mathrm{~mm}$ thick from $\mathrm{c}$ to d) $I$ is relatively low and uniform. No anatomical layers can be clearly differentiated. In contrast, $Q, U$, and $V$ images of the cornea are nonuniform: the polarization state of backreflected light varies as a function of depth and lateral position in the cornea. Variations of $Q, U$, and $V$ values in depth in the corneal stroma are due to the cumulative polarization effect of all lamellae. Each lamella acts as linear retarder.

A prominent feature indicating an increase in the $V$ value (from black to white) is observed in the right half of the cornea on the $V$ image, meaning polarization of backreflected light changes from left circular to approximately right circular. Therefore, in this region, the whole corneal stroma acts as a quarter-wave retarder in single pass (half-wave retarder in double pass). A different feature is observed in the left half of the $V$ image. There, backscattered light polarization state changes from left circular at the glass-cornea interface (point c) to approximately linear at $-45^{\circ}(U \sim-1)$ at the cornea-aqueous interface (point d). Therefore, the cornea birefringence varies between the left and right side of the imaged region.

In the cornea we also observe that the $Q$ parameter value decreases (from gray to dark pixels) with depth for given lateral position and increases (from gray to bright pixels) with depth at other lateral position. This can be explained only by variations in the local optic axis direction as a function of lateral position. Indeed, if the optic axis direction was constant over the imaged region, the $Q$ parameter would vary monotonically as a function of depth but could not increase and decrease.

Our results are consistent with the observations of Chang et al. [25] using small angle light scattering. They found that the local birefringence and optic axis of the rabbit cornea depends on lateral position. The glass slide modified the anatomical structure of the cornea and may have induced stress birefringence. However, we expect the effect was small, inasmuch as we observed similar local variations in polarization state of backscattered light from corneas that were unstressed. In the $Q, U$, and $V$ images we see that the polarization state of light is not modified by the anterior chamber fluid and remains constant in the anterior lens. Therefore, the aqueous fluid and lens do not appear birefringent.

2) A-Scans Averages: The amplitude of the Stokes parameters as a function of depth, i.e., in A-scan mode, has a significant noise component due to speckle effect, detector shot noise and electronic amplification noise. To decrease this noise we averaged laterally adjacent pixel values (after reregistering A-scans depth positions to align laterally on the glass slide surface). Fig. 4 shows an example of an average of 25 adjacent A-scans for the intensity $I$ and phase retardation $\Phi$ calculated according to (5). The left $y$ axis corresponds to $I$ in $\mathrm{dB}$ (solid line) and the right $y$ axis refers to $\Phi$ in degrees (dashed line). The letters above the $x$ axis refer to the depth positions described in Fig. 3. In the air (a-b), glass $(b-c)$, and aqueous (d-e) the intensity $I$ is minimum. $I$ peaks at the b) air-glass, c) glass-cornea, d) cornea-aqueous, and e) aqueous-crystalline lens interfaces. In the cornea (c-d), $I$ varies between -70 and $-80 \mathrm{~dB}$, and in the anterior part of the crystalline lens it decreases slowly down to the noise level $(-92 \mathrm{~dB})$. In air $(\mathrm{a}-\mathrm{b})$, glass $(\mathrm{b}-\mathrm{c})$, and aqueous $(\mathrm{d}-\mathrm{e})$ the backreflected light intensity is below the noise level of the PSOCT system. In 

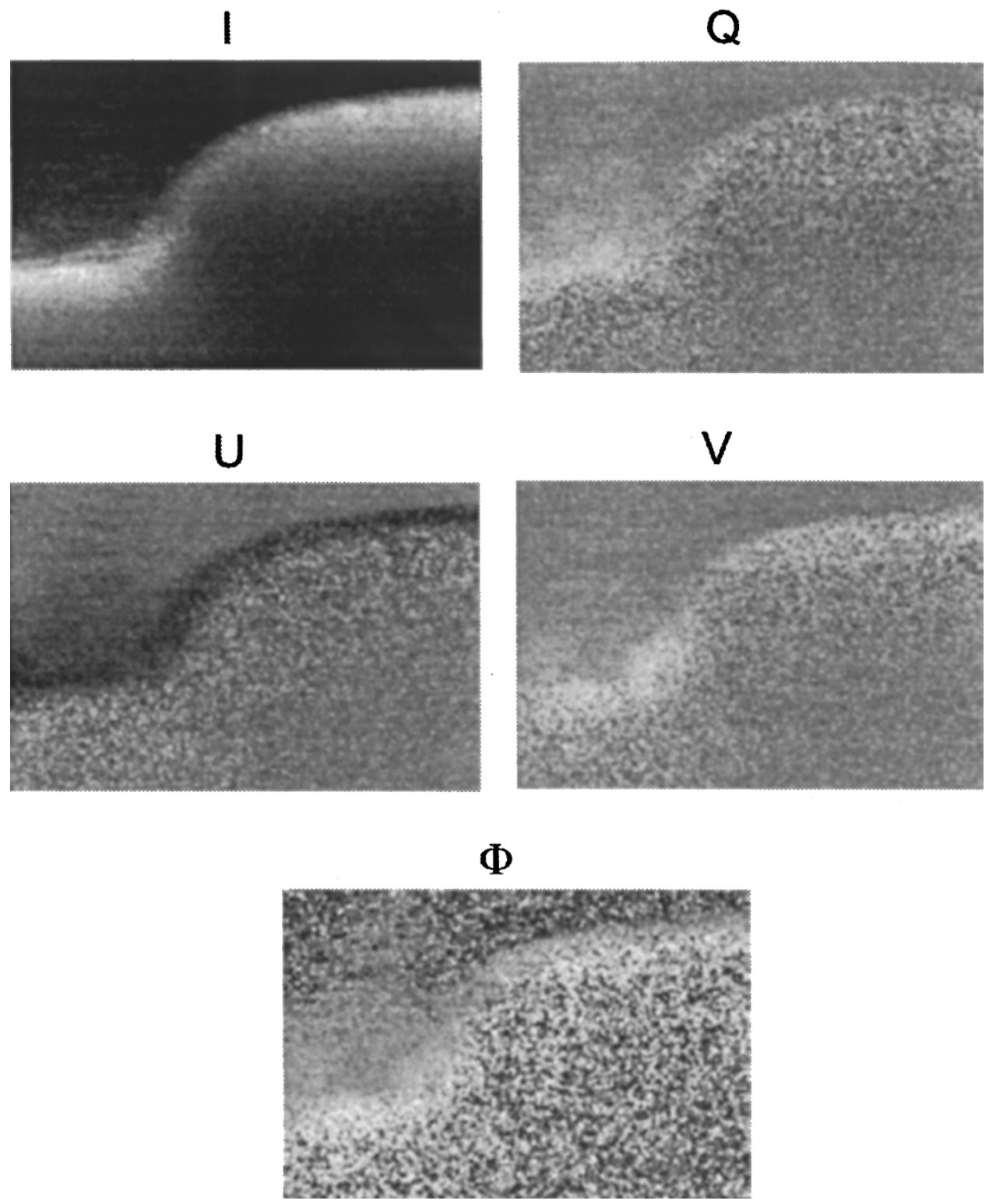

Fig. 5. $I, Q, U$, and $V$ Stokes parameters and phase retardation $\Phi$ images in the rabbit retina. The left edge is approximately at the center of the optic nerve. A significant change in phase retardation can be observed on the $\Phi$ image at the position of the NFL.

these regions, the value of $\Phi$ is not related to polarization state of the backreflected light but varies randomly between $0^{\circ}$ and $180^{\circ}$ on individual A-scans. $\Phi$ averages $65^{\circ}$ in Fig. 4 , instead of $90^{\circ}$ (all values have equal probability), probably because of an imbalance in the detectors noise figure. At the air-glass b), glass-cornea c), and aqueous-crystalline lens e) interfaces, we observed that when the intensity increases by 6 $\mathrm{dB}$ above the noise level, $\Phi$ varies by more than $70 \%$ of its initial value. Therefore we decided to consider that $\Phi$ value was representative of the polarization state of the backreflected light when the intensity $I$ is $6 \mathrm{~dB}$ or more above the noise level ( $86 \mathrm{~dB}$ in Fig. 4).

In the cornea, $\Phi$ increases from $0^{\circ}$ to about $120^{\circ}$, indicating that the polarization state of the backreflected light varies from left circular to some right elliptical state between the anterior and posterior side of the cornea. The aqueous does not modify the phase retardation, since $\Phi$ equals $120^{\circ}$ at the posterior cornea and anterior crystalline lens. Deeper in the lens $\Phi$ decreases. The value of $\Phi$ computed from pixels $250-350$ is unreliable because the intensity is lower than $-86 \mathrm{~dB}$.

\section{B PSOCT Imaging in the Retina}

1) Stokes Parameter Images: The $I, Q, U$, and $V$ Stokes parameter images acquired in the retina of an enucleated rabbit eye, as well as the phase retardation $\Phi$ calculated with (5), are presented in Fig. 5. To reduce speckle noise, each pixel value in the $Q, U$, and $V$ images was multiplied by the $I$ image pixel value, a low-pass filter was applied to the resulting image, and each pixel was divided by the local average intensity. Each image represents a $3 \mathrm{~mm}$ wide $\times 2 \mathrm{~mm}$ deep section. The left edge corresponds approximately to the center of the optic nerve. Above the optic nerve the posterior hyaloid phase backscatters some light and shows up as bright and dark "clouds" in the $I$ and $U$ images, respectively. The dark 


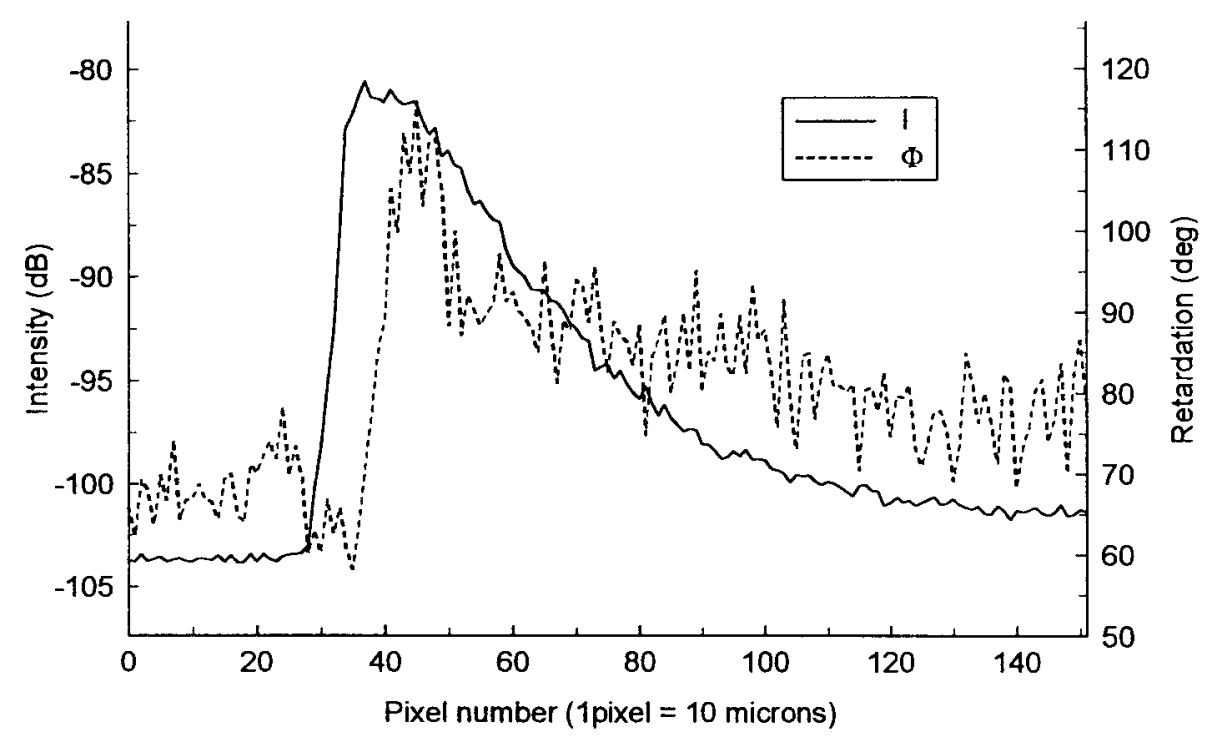

Fig. 6. The values of $I$ in $\mathrm{dB}$ (solid line) and $\Phi$ in degree (dashed line) are plotted as a function of depth in the rabbit retina. Each plot is an average of 50 depth scans, corresponding to a lateral scanning distance of $500 \mu \mathrm{m}$. The intensity $I$, i.e., the conventional OCT signal, increases from -104 to $-81 \mathrm{~dB}$ at the vitreous-retina interface. The retardation $\Phi$, calculated with (5), increases from about $60^{\circ}$ to $115^{\circ}$ over a depth of $90 \mu \mathrm{m}$.

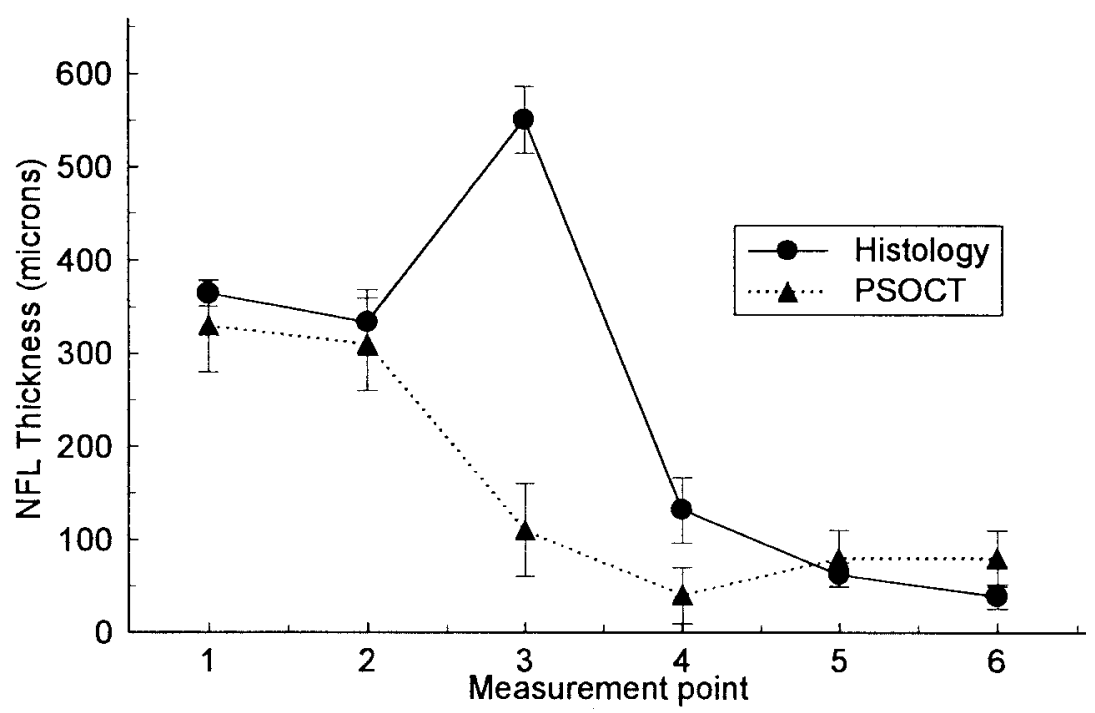

Fig. 7. Comparison of NFL thickness measurement in histology section and with PSOCT. The distance between successive measurement points is 500 $\mu \mathrm{m}$. Point 1 is approximately at the center of the optic nerve.

and light bands following the contour of the retina in the $U$ and $V$ images, respectively, mean that the light backreflected from this region is an elliptical polarization state between right circular and linear at $-45^{\circ}$. In the $\Phi$ image, we notice a region on top of the retina (right half) and in the optic nerve (left third) that changes approximately from gray to white as a function of depth, corresponding to an increase in phase retardation. In the region where the retina curves into the optic nerve head this increase is not as pronounced. At these positions, the axons are oriented downward, and the optic axis of the tissue is nearly parallel to the incident light propagation direction.

2) A-Scans Averages: Plots of $I$ and $\Phi$ as a function of depth in the retina were obtained by averaging 50 laterally adjacent A-scans. An example is presented in Fig. 6. The intensity increases from -104 to about $-81 \mathrm{~dB}$ at the vitreousretina interface, is maintained at $-81 \mathrm{~dB}$ over approximately
$100 \mu \mathrm{m}$, then decreases exponentially to $-102 \mathrm{~dB}$ in $1 \mathrm{~mm}$. Between pixels 31 and 90 the intensity is greater than $6 \mathrm{~dB}$ above the noise level $(-102 \mathrm{~dB})$. In this region, $\Phi$ describes reliably the polarization state of the backreflected light. At the vitreous-retina interface the intensity $I$ increases sharply and $\Phi$ drops to about $60^{\circ}$. Although noise is observed in the $\Phi$ plot, the increase after the vitreous-retina interface is significantly higher than the noise amplitude. The distance between the minimum and maximum of the phase retardation was measured on a smoothed $\Phi$ plot (a low-pass or median filter can be used). For example, in Fig. 6, $\Phi$ increased over a depth of $90 \mu \mathrm{m}$ (pixels 35-44). Since the NFL is the only known birefringent layer in the retina we believe the region representing a significant change in phase retardation corresponds to the NFL. The shape of the $\Phi$ plot versus depth depends on the birefringence and optic axis orientation of the 
tissue as well as on the polarization state of light incident on the retina. In the worst case, light incident on the retina is linearly polarized parallel to the optic axis of the NFL and no change in any of the Stokes parameters is observed. Although the chances of this happening appear remote, the problem could be resolved by probing the eye with more than one incident polarization states.

3) Comparison with Histology: NFL thickness was measured on histology slides every $500 \mu \mathrm{m}$ between the black ink dots indicating the boundaries of the PSOCT-imaged region. The depth-resolved phase retardation and intensity plots were calculated at the corresponding positions by averaging 50 adjacent A-scans $(500 \mu \mathrm{m})$. The thickness of the region presenting a significant change in phase retardation was measured with the method described in the previous section. Fig. 7 shows NFL thickness as determined by histology and PSOCT as a function of lateral position. Six points positioned $500 \mu \mathrm{m}$ apart were considered. Point 1 corresponds approximately to the center of the optic nerve. The optic nerve diameter was approximately $2 \mathrm{~mm}$. The optic nerve margin was near point 3. Good correspondence is observed between NFL thickness measured by both methods except for points 3 and possibly 4. At point 3 , the NFL curves into the optic nerve and the measurement on the histology section contains an artifact due to the position and orientation of the section. A birefringent layer was observed for points 1 and 2 that are inside the optic nerve head. This is consistent with the histology results and can be explained by the fact that the imaged section does not pass through the center of the optic nerve where the birefringence should be zero, since all fibers (and thus the tissue optic axis) are parallel to the illumination beam. With SLP and OCT, NFL thickness is typically measured in a cylindrical ring around the optic nerve with a diameter of 1.5-1.75 optic discs, corresponding to the location of points 5 and 6 in our experiment. At these points, NFL thickness measured by PSOCT is in relatively good agreement with the histological results.

\section{CONCLUSION}

Depth-resolved Stokes parameter images of the corneas and retinas in New Zealand white rabbit eyes were acquired with PSOCT. We demonstrated that the cornea is birefringent and that the birefringence as well as the optic axis of the stroma vary as a function of lateral position. PSOCT could be used to study the corneal stroma structure more extensively. With a good depth resolution (limited only by the source bandwidth and dispersion unbalance in the interferometer) microscopic changes in the polarization state of the light may be observed. With the techniques currently used to study the stromal structure [2], the cornea has to be excised. In our experiments, the cornea was still attached to the eye. With our current system, we can only perform in vitro imaging. However the PSOCT system can be modified to reduce image acquisition time and avoid motion artifacts allowing for in vivo imaging.

We also imaged the retina and observed a birefringent layer at the vitreous-retina interface. The local thickness of the birefringent layer determined using PSOCT corresponded well to the thickness of the NFL measured from histology slides in four out of six considered points. Further studies are required to evaluate the reliability of the NFL thickness measurement with PSOCT. Modifications in the apparatus and signal processing are being investigated to increase the lateral and depth resolution, reduce speckle noise, and increase NFL contrast in PSOCT images.

\section{REFERENCES}

[1] A. Stanworth, "Polarized light studies of the cornea," J. Exp. Biol., vol. 30, pp. 164-169, 1953.

[2] W. A. Christens-Barry, W. J. Green, P. J. Connolly, R. A. Farrel, and R. L. McCally, "Spatial mapping of polarized light transmission in the central rabbit cornea," Exp. Eye Res., vol. 62, pp. 651-662, 1996.

[3] D. J. Donohue, B. J. Stoyanov, R. L. McCally, and R. A. Farrel, "A numerical test of the normal incidence model of corneal birefringence," Cornea, vol. 15, pp. 278-285, 1996.

[4] _ "Numerical modeling of the cornea's lamellar structure and birefringence properties," J. Opt. Soc. Amer. A, vol. 12, pp. 1425-1438, 1995.

[5] R. Varma, M. Skaf, and E. Barron, "Retinal nerve fiber layer thickness in normal human eyes," Ophthalmology, vol. 103, 1996.

[6] R. P. Hemenger, "Birefringence of a medium of tenuous parallel cylinders," Appl. Opt., vol. 28, pp. 4030-4034, 1989.

[7] Q. Zhou and R. W. Knighton, "Light scattering and form birefringence of parallel cylindrical arrays that represent cellular organelles of the retinal nerve fiber layer," Appl. Opt., vol. 36, 1997.

[8] H. A. Quigley E. M. Addicks, and W. R. Green, "Optic nerve damage in human glaucoma-III: Quantitative correlation of nerve fiber loss and visual field defect in glaucoma, ischemic neuropathy, papilledema, and toxic neuropathy," Arch. Ophthalmol., vol. 100, pp. 135-146, 1982.

[9] J. S. Schuman, T. Pedut-Kloizman, E. Hertzmark, M. R. Hee, J. R. Wilkins, J. G. Coker, C. A. Pufialito, J. G. Fujimoto, and E. A. Swanson, "Reproducibility of nerve fiber layer thickness measurements using optical coherence tomography," Ophthalmology, vol. 103, 1996.

[10] N. T. Choplin, D. C. Lundy, and A. W. Dreher, "Differentiating patients with glaucoma from glaucoma suspects and normal subjects by nerve fiber layer assessment with scanning laser polarimetry," Ophthalmol., vol. 105, 1998.

[11] R. C. Youngquist, S. Carr, and D. E. N. Davies, "Optical coherencedomain reflectometry: A new optical evaluation technique," Opt. Lett., vol. 12, pp. 158-160, 1987.

[12] A. F. Fercher, "Optical coherence tomography," J. Biomed. Opt., vol. $1,1996$.

[13] C. A. Toth, D. G. Narayan, S. A. Boppart, M. R. Hee, J. G. Fujimoto, R. Birngruber, C. P. Cain, C. D. DiCarlo, and W. P. Roach, "A comparison of retinal morphology viewed by optical coherence tomography and light microscopy," Arch. Ophthalmol., vol. 115, 1997.

[14] R. N. Weinreb, "Histopathologic validation of the Fourier-ellipsometry measurements of the retinal nerve fiber layer thickness," Arch. Ophthalmol., vol. 108, pp. 557-560, 1990.

[15] A. W. Dreher, K. Reiter, and R. N. Weinreb, "Spatially resolved birefringence of the retinal nerve fiber layer assessed with a retinal laser ellipsometer," Appl. Opt., vol. 31, 1992.

[16] R. N. Weinreb, L. Zangwill, C. C. Berry, R. Bathija, and P. A. Sample, "Detection of glaucoma with scanning laser polarimetry," Arch. Ophthalmol., vol. 116, pp. 1583-1589, 1998.

[17] L. Zangwill, C. A. Berry, V. S. Garden, and R. N. Weinreb, "Reproducibility of retardation measurements with the nerve fiber analyzer II," J. Glaucoma, vol. 6, pp. 384-389, 1997.

[18] M. J. Tjon-Fo-Sang, "Measurement by nerve fiber analyzer of retinal nerve fiber layer thickness in normal subjects and patients with ocular hypertension," Amer. J. Ophthalmol., vol. 122, pp. 220-227, 1996.

[19] J. F. de Boer, T. E. Milner, M. J. C. van Gemert, and J. S. Nelson, "Two-dimensional birefringence imaging in biological tissue by polarization-sensitive optical coherence tomography," Opt. Lett., vol. 22, pp. 934-936, 1997.

[20] M. J. Everett, K. Schoenenberger, B. W. Colston, and L. B. Da Silva, "Birefringence characterization of biological tissue by use of optical coherence tomography," Opt. Lett., vol. 23, pp. 228-230, 1998.

[21] J. F. de Boer, S. M. Srinivas, A. Malekafzali, Z. Chen, and J. S. Nelson, "Imaging thermally damaged tissue by polarization sensitive optical coherence tomography," Optics Express., vol. 3, pp. 212-218, 1998. 
[22] K. Schoenenberger, B. W. Colston, D. J. Maitland, L. B. Da Silva, and M. J. Everett, "Mapping of birefringence and thermal damage in tissue by use of polarization-sensitive optical coherence tomography," Appl. Opt., vol. 37, pp. 6026-6036, 1998.

[23] J. F. de Boer, T. E. Milner, and J. S. Nelson, "Determination of the depth resolved stokes parameters of light backscattered from turbid media using polarization sensitive optical coherence tomography," Opt. Lett., vol. 24, pp. 300-302, 1999.

[24] W. A. Shurcliff and S. S. Ballard, Polarized Light. New York: D. Van Nostrand, 1964.

[25] E. P. Chang, D. A. Keedy, and J. C. W. Chien, "Ultrastructures of rabbit corneal stroma: Mapping of optical and morphological anisotropies," Biochimica et Biophysica Acta, vol. 343, pp. 615-626, 1974.

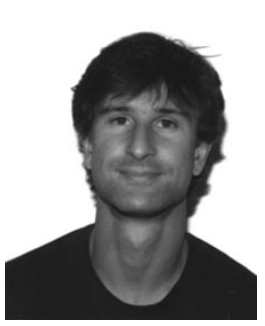

Mathieu G. Ducros received the Engineering Diploma from Ecole Centrale de Lille, France, in 1995, and the M.S. degree from the University of Texas at Austin in 1996. Since 1995, he has been studying biomedical engineering and specializing in biomedical optics. He is currently a Ph.D. candidate in the Biomedical Engineering Program, University of Texas at Austin.

His main research interests are optical diagnostic and treatment methods in ophthalmology and dermatology. He is a member of the Optical Society of America.

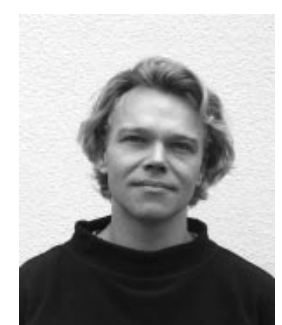

Johannes F. de Boer received the B.S., M.S., and Ph.D. degrees in solid state physics from the University of Amsterdam, The Netherlands.

The subject of the Ph.D. dissertation was the interference effects of multiple scattered light in disordered structures. In 1995, he joined the Academic Medical Center in Amsterdam as a Post-Doctoral Fellow, where he became interested in polarization effects in Optical Coherence Tomography. In 1997, he joined the Beckman Laser Institute and Medical Clinic, University of California at Irvine, where he has been an Assistant Professor since 1998.
Huai-En Huang, photograph and biography not available at the time of publication.

Lawrence C. Chao, photograph and biography not available at the time of publication.

Zhongping Chen, for photograph and biography, see this issue, p. 1141.

J. Stuart Nelson, for photograph and biography, see this issue, p. 1066.

Thomas E. Milner, for photograph and biography, see this issue, p. 1066.

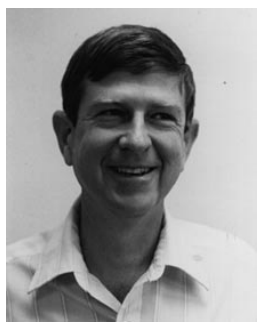

H. Grady Rylander, III (S67-A'75) received the B.S.E.E. and M.S.E.E. degrees from the University of Texas at Austin in 1970 and 1974, respectively, and the M.D. degree from the University of Texas Health Science Center at San Antonio in 1974.

He has practiced ophthalmology and taught at the University of Texas at Austin since 1974, where he is a Professor in the Department of Electrical and Computer Engineering and in the Biomedical Engineering Program. Research interests include laser-tissue interaction, robotic vision, signal processing, RF effects on the eye, and neuroprosthesis.

Dr. Rylander is a member of Eta Kappa $\mathrm{Nu}$, Tau Beta Pi, a Fellow of the American Academy of Ophthalmology, and is a Registered Professional Engineer in the State of Texas. 